\title{
Evolution and Optimization of Active Queue Management Algorithms over High Bandwidth Aggregates
}

\author{
Saurabh Sarkar \\ Department of CSE \\ Dr. B.R.Ambedkar, NIT \\ Jalandhar, India
}

\author{
Geeta Sikka \\ Department of CSE \\ Dr. B.R.Ambedkar, NIT \\ Jalandhar, India
}

\author{
Ashish Kumar \\ Department of CSE \\ Dr. B.R.Ambedkar, NIT \\ Jalandhar, India
}

\begin{abstract}
Due to enormous growth of the Internet, demands for access from multiple users and request for new services for its applications have also increased significantly. In a result there is a rise in packet loss rates and drop in network efficiency. In addition, the inability to support new services has severely hindered the widespread deployment of bandwidth-sensitive applications. So the success of TCP / IP depends on its ability to deliver services in time of extremely high demand and hence various congestion control mechanisms ensure this ability to prosper. The idea behind TCP congestion control is to control network load by having sources adjust their rates according to the level of congestion in the network. This paper focuses on how congestion control and queue management techniques have evolved in due course of time and being modified to minimize the rate of packet loss. Considering RED being most important of all we have optimized the algorithm by challenging the linearity of marking/dropping probability. We conduct a survey by applying different possible functions in contrary to the linear behavior of dropping probability for evaluating their performance in comparison to RED. We implement the optimized RED by simulating a multicast network for TCP flows.
\end{abstract}

\section{General Terms}

Active Queue Management Algorithms, RED, Non-linear model of RED.

\section{Keywords}

AQM, RED, drop probability, simulations, NS2.

\section{INTRODUCTION}

Various connections in a network compete for resources like link bandwidth and buffer space in the router during network congestion. One of the conventional solutions to congestion is using the window-based TCP congestion control at the endhosts. The Internet Engineering Task Force (IETF) considered the deployment of Explicit Congestion Notification (ECN) [1] and later on advances in active queue management [2] as a means to reduce packet loss. Initially ECN was a success in effectively reducing the transmission rates of individual connection during congestion; but it does not provide a complete solution to the congestion problem of the network as a whole. This is because TCP sources reduce their transmission rates only after detecting packet loss caused by queue overflow at the router. It takes some time between packet being dropped at the router and its detection at the source during which a large number of packets may have been continuously being dropped while the sources continue to transmit at a rate that the network cannot support.

On the other hand AQM schemes have been proposed to complement the TCP network congestion control which helps in detecting congestion early dropping packets randomly before the buffer becomes completely full. The ability of $\mathrm{AQM}$ to detect incipient congestion and convey congestion notification to the end-hosts enables the sources to reduce their sending rates prior to buffer overflow. ECN is used in conjunction with AQM for signaling congestion to sources using packet marking instead of dropping packets.

\section{QUEUING ALGORITHMS}

Various queuing algorithms are implemented in a network router to obtain desired bandwidth. The simplest type of queue transmits packets in the same order that it receives them. This is called a First in First out (FIFO) queue. Generally it may seem that it treats all the traffic streams equally, but it actually tends to favor resource-hungry, illbehaved applications. The problem is that if a single application sends a burst that fills a FIFO queue, the router will wind up transmitting most of the queued packets, but will have to drop incoming packets from other applications. If these other applications adapt to the decrease in available bandwidth by sending at a slower rate, then the ill-behaved application will greedily take up the slack and could gradually choke of all the other applications.

Because FIFO queuing allows some data flows to take more than their share of the available bandwidth, it is called unfair. Fair Queuing (FQ) and Weighted Fair Queuing (WFQ) are two of the simpler algorithms that have been developed to deal with this problem. Both of these algorithms sort incoming packets into a series of flows.

Following are the few existing queuing algorithms available for our network routers [3]:

- Drop Tail is a queuing algorithm based on a firstcome-first-serve discipline.

- Fair Queuing is an algorithm that attempts to allocate bandwidth fairly among all input flows.

- Stochastic Fair Queuing involves a hash function used to map flows to a queue.

- Deficit Round Robin scheduling is an algorithm that services each flow in a predefined sequence.

- Random Early Detection (Drop) tries to anticipate congestion by monitoring the queue. When the 
specified threshold is reached, it randomly discards or marks the packets. This is the most widely accepted algorithm till date.

- Class Based Queuing (Packet by Packet Round Robin and Weighted Round Robin) queues packets according to criteria defined by an administrator. It provides differential forwarding service for each class. Packets are divided into a hierarchy of classes defined by input flows.

\section{DIFFERENT CLASSES OF ACTIVE QUEUE MANAGEMENT ALGORITHMS}

In this section we will discuss few important AQM algorithms that are being proposed over the last decade. The performance of the TCP congestion control mechanism in networks that implement drop-tail [3] packet discard has some drawbacks, such as synchronization of flows, inequitable distribution of packet loss among flows, and low utilization of network resources. Therefore, even with end systems equipped with important algorithms such as the TCP congestion avoidance, slow start, fast retransmit, and fast recovery mechanisms [1]' the performance of the TCP congestion control algorithm over current drop-tail networks can still be unsatisfactory. Active queue management has been recommended by the Internet Engineering Task Force (IETF) as a way of mitigating the above stated performance limitations of TCP over drop-tail networks. Random Early Detection (RED) [4] is the first active queue management algorithm proposed for deployment in TCP/IP networks. The basic idea behind an active queue management algorithm is to convey congestion notification early to the TCP end-points so that they can reduce their transmission rates before queue overflow and sustained packet loss occur. It is now widely accepted that a RED controlled queue performs better than a drop-tail queue. However, RED has some parameter tuning issues that need to be carefully addressed for it to give good performance under different network scenarios. As a result several algorithms, like BLUE [5] and Stabilized RED (SRED) [5]' have been developed as alternatives to RED. Although these algorithms also control congestion by discarding packets with a load-dependent probability whenever a queue in the network appears to be congested, they are designed with the objective of maintaining stabilized network queues, thereby minimizing occurrences of queue overflows and underflows, and providing high system utilization. The Dynamic RED (DRED) active queue management algorithm [5] also has similar objectives. However, unlike SRED and BLUE, this new active queue management algorithm uses a simple feedback control approach to compute the drop probabilities used to discard packets during times of queue congestion.

Although these AQM algorithms are highly robust to diverse network conditions, most of them were designed without considering their robustness against network attacks, such as the Denial-of-Service (DoS) [6,7] attacks that have been identified as a major threat to today's Internet services. Example DoS attacks include TCP SYN attacks, ICMP directed broadcasts and DNS flood attacks. These attacks normally generate high rate transmission of packets toward the victim node. They can be detected and alleviated [3,6,7]. Recently a new kind of DoS attack, low-rate DoS attack, has been proposed in that exploits TCP's retransmission timeout mechanism to reduce TCP throughput without being detected. Compared to traditional flooding based DoS attacks, the lowrate DoS attack does not employ a "sledge-hammer" approach of high-rate transmission of packets, and consequently eludes detection. RED-like algorithms have already been found to be notably vulnerable to LDoS attacks.

For this a novel Robust RED (RRED) algorithm [8] is proposed to thwart the LDoS attacks. The RRED algorithm consists of a new detection algorithm and a traditional RED algorithm. The basic idea behind the RRED is to detect and filter out LDoS attack packets from incoming flows before they feed to the RED algorithm. Bloom filter techniques have been used in the implementation of the RRED algorithm to manage potentially numerous incoming flows and increase the detection accuracy. Experiment results show that the RRED algorithm is highly robust when the router is under an LDoS attack. The TCP traffic remains its ideal rate and TCP throughput is nearly fully preserved.

\section{OPTIMIZATION OF RANDOM EARLY DETECTION USING A NON- LINEAR MODEL FOR DROPPING PACKETS}

Understanding AQM congestion control requires knowledge on the response characteristics of the traffic to control. Internet traffic has been dominated by TCP that responds to network congestion in an Additive Increase Multiplicative Decrease (AIMD) manner [9]. We have used a non- linear model for dropping packet rather than linear model which is used in the conventional RED scheme. The non linear model explained later in this section is particularly useful for configuring the Random Early Detect ion (RED) family router queue management mechanisms that use the average queue length to compute drop probability.

We choose to study and evaluate RED-family AQM for the several reasons: First, while recently proposed AQM mechanisms such as RRED [8], DRED [5], and BLUE [5] do not strictly use the average queue to compute congestion, they have performance goals similar to that of RED- family AQMs. Second, since RED congestion control mechanisms are relatively well-understood and are commonly used as a benchmark for evaluation of other AQM mechanisms, further extending an understanding of RED-family mechanisms and illustrating possible outcomes of RED will help researchers to design experiments that fairly compare RED with other AQM mechanisms. Third, with the help of a general modification, it is easy to configure RED- family AQMs to create various test scenarios that reveal interesting AQM congestion control issues. In turn, a general non- linear model proposed can be revalidated through evaluation with different configurations presented in RED family. Lastly, since RED is already implemented in some commercial routers, our optimized RED can be used to tune these routers, thus realizing some of the potential benefits of ECN with few network infrastructure changes.

RED maintains an exponentially weighted moving average (EWMA) of the queue length which it uses to detect congestion. RED detects increases in the average queue length and uses it to determine whether or not to drop or ECN -mark a packet. More specifically, Fig 1 plots the marking/dropping probability of RED as a function of the average queue length. As the figure shows, when the average queue length exceeds a minimum threshold $\left(\mathrm{th}_{\mathrm{min}}\right)$, packets are randomly dropped or marked with a given probability. A connection receiving congestion notification in the form of an ECN mark cuts its congestion window in half as it would if it had detected a packet loss. The probability that a packet arriving at the RED queue is either dropped or marked depends on, among other 
things, the average queue length and an initial probability parameter $\left(\mathrm{P}_{\max }\right)$. As Fig 1 shows, the calculated marking/dropping probability is a linear function of the average queue length. The probability is 0 when the average queue length is less than or equal to $t_{\min }$ and linearly increases $\mathrm{P}_{\max }$ when the average queue length approaches a maximum threshold $\left(\mathrm{th}_{\max }\right)$. If average queue length exceeds $\mathrm{th}_{\max }$, all packets are dropped or marked.

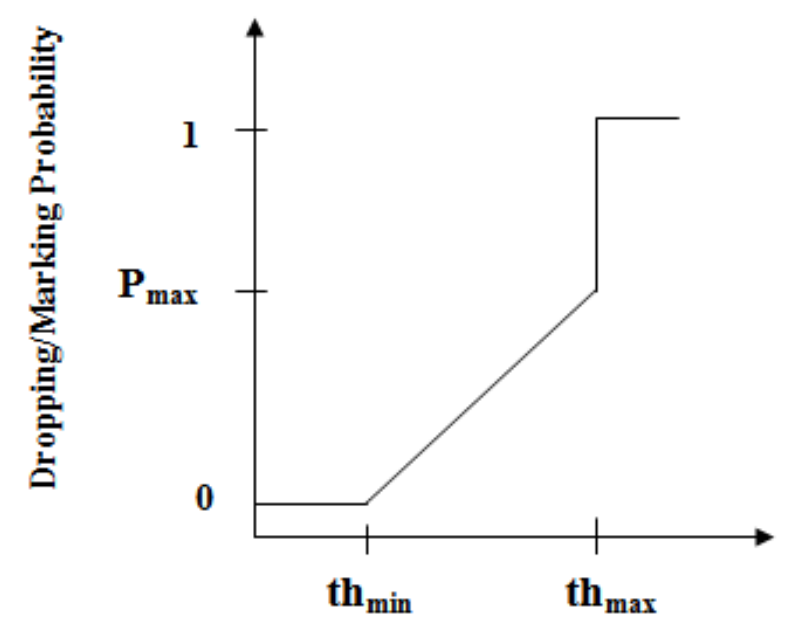

\section{Average Queue Length}

Fig 1: The marking/dropping behavior of RED.

The general RED algorithm [4] is given below (calculate the average queue size avg):-.

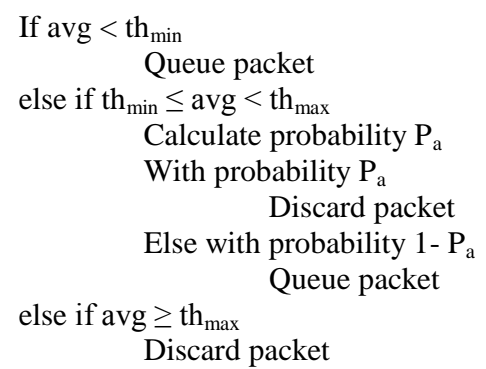

\subsection{Average Queue Size}

The average queue size is calculated using an exponentially weighted average (EWA) of previous queue lengths.

$$
\begin{array}{cl}
\text { If } \mathrm{q}>0 & \\
& \text { avg }=\left(1-\mathrm{w}_{\mathrm{q}}\right) \operatorname{avg}+\mathrm{w}_{\mathrm{q}} \mathrm{q} \\
\text { else } & \mathrm{t}=\mathrm{f}\left(\text { time }-\mathrm{q}_{\text {_time }}\right) \\
& \operatorname{avg}=\left(1-\mathrm{w}_{\mathrm{q}}\right)^{\mathrm{t}} \text { avg }
\end{array}
$$

The weight $\mathrm{w}_{\mathrm{q}}$ determines how rapidly avg changes in response to changes in actual queue size. The number $t$ is estimated by the router's idle time; in this period $\mathrm{m}$ packets could have been transmitted. With $\mathrm{w}_{\mathrm{q}}$ the algorithm can avoid reacting to short bursts of congestion.

\subsection{Probability of Discard}

Let $\mathrm{c}$ be the number of how many consecutive packets have not been discarded since the last packet was dropped. It is obvious that the higher the value of $\mathrm{c}$, the higher the probability of discard. Let $\mathrm{P}_{\mathrm{b}}$ represent a temporary probability varies from 0 at avg $=\mathrm{th}_{\min }$ to $\mathrm{P}_{\max }$ at avg $=\mathrm{th}_{\min }$.

$$
\begin{gathered}
P_{b}=P_{\text {max }}\left(a v g-t h_{\text {min }}\right)\left(t h_{\text {max }}-t h_{\text {min }}\right) \\
P_{a}=P_{b} /\left(1-c \times P_{b}\right)
\end{gathered}
$$

When $\mathrm{c}=1 / \mathrm{P}_{\mathrm{b}}-1, \mathrm{P}_{\mathrm{a}}=1$. But $\mathrm{P}_{\mathrm{a}}$ increases very slowly when $\mathrm{c}$ is not very big. Only when $\mathrm{c}$ is close to $1 / \mathrm{P}_{\mathrm{b}}-1, \mathrm{P}_{\mathrm{a}}$ begins to rise dramatically.

\section{SIMULATION SCENARIOS AND EVALUATION}

In this part, we modified the equation in step (1) by applying two different functions (viz. V1 and V2) to calculate drop probability $\left(\mathrm{P}_{\mathrm{b}}\right)$ and compare the results. The curves for the two functions V1 \& V2 can be assumed roughly by Fig 2:-

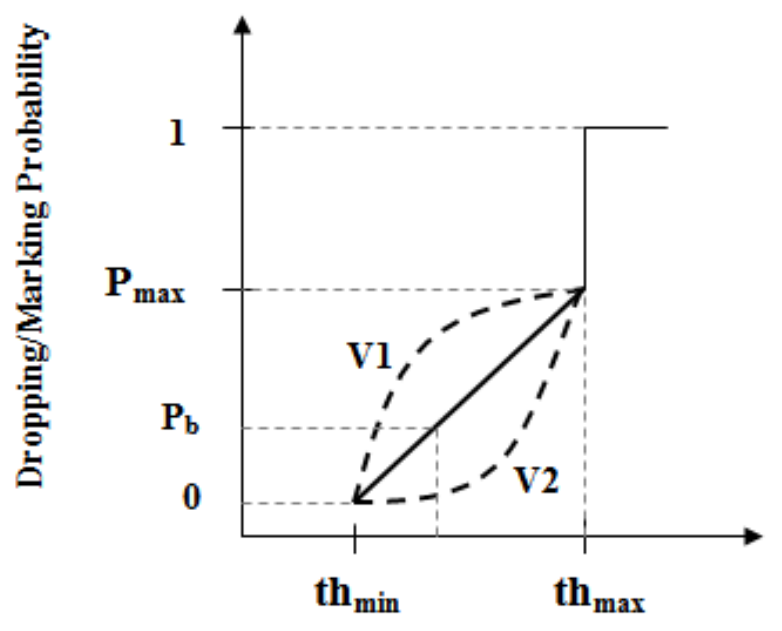

Average Queue Length

Fig 2: Assumed behavior of RED

In NS2 RED queue implementation doesn't use the formula (1) above directly, instead it uses the formula below,

$$
P_{b}=P_{\max } \times\left(v_{-} a \times v_{-} a v e+v_{-} b\right)
$$

where $\mathrm{v} \_\mathrm{a}=1.0 /\left(\mathrm{th}_{\max }-\mathrm{th}_{\min }\right)$, $\mathrm{v} \_$ave is the average queue size, and $v_{-} b=-t_{\min } /\left(\mathrm{th}_{\max }-\mathrm{th}_{\min }\right)$. This forumla is equivalent to formula (1).

Now considering equation (3) we can see that drop probability $\left(\mathrm{P}_{\mathrm{b}}\right)$ linearly depend upon the average queue size i.e. $\mathrm{v} \_$ave. In order to increase or decrease the drop probability as shown in Fig 2 we need a non-linear dependency of $\mathrm{P}_{\mathrm{b}}$ upon $\mathrm{v} \_$ave.

For increasing the drop probability than usual we need a concave function (V1) instead of a linear function as in (3). In our simulation we have considered $\log$ as the concave function (V1) applied to transform the formula (3) in following form:-

$$
P_{b}=P_{\max } \times\left(m \times \log \left(v_{-} a v e\right)+n\right)
$$

Hence $m$ and $n$ are constants to be derived with respect to $\mathrm{v} \_\mathrm{a}^{\mathrm{a}}$ and $v_{-} b$.

As described in Fig $\mathbf{2}$ the curve intersects with original linear function at $\mathrm{th}_{\min }$ and $\mathrm{th}_{\max }$, so we can say that the curve $\mathrm{V} 1$ satisfies the points $\left(\mathrm{th}_{\min }, 0\right)$ and $\left(\mathrm{th}_{\max }, \mathrm{P}_{\max }\right)$. 
Hence by satisfying these points in equation (4) we have the following,

$$
\begin{aligned}
& 0=P_{\text {max }} \times\left(m \times \log \left(t h_{\text {min }}\right)+n\right. \\
& P_{\text {max }}=P_{\text {max }} \times\left(m \times \log \left(t h_{\text {max }}\right)+n\right.
\end{aligned}
$$

Also the original linear function gives us the function,

$$
0=P_{\max } \times\left(v_{-} a \times t h_{\text {min }}+v_{-} b\right)
$$

with (5) (6) and (7), we can get values for $\mathrm{m}$ and $\mathrm{n}$ in formula (4),

$$
\begin{gathered}
m=\frac{1}{\left(\log \left(t h_{\max }\right)-\log \left(-v_{-} b / v_{-} a\right)\right)} \\
n=-m \times \log \left(-v_{-} b / v_{-} a\right)
\end{gathered}
$$

Similarly, we can assume a decrease in drop probability as shown in Fig 2 (V2) using a convex function. In our simulation we have considered $x^{2}$ as the convex function (V2), which is again applied to transform the formula (3) in following form :-

$$
P b=P_{\max } \times\left(m \times v_{-} a v e^{2}+n\right)
$$

By obtaining the value of the constants $m$ and $n$ in similar way, we have:-

$$
\begin{aligned}
& m=\frac{1}{\left(t h_{\max }{ }^{2}-\left(-v_{-} b / v_{-} a\right)^{2}\right)} \\
& n=-m \times\left(-v_{-} b / v_{-} a\right)^{2}
\end{aligned}
$$

\subsection{Simulation Configuration}

Considering the scenario discussed in the previous section we configured and run the related NS-2 program to get simulation results. The results are analyzed by several perl and python programs and the figures are drawn by gnuplot. The configuration of this simulation is with reference to the problem defined in [10]:

\subsubsection{Network Topology}

Fig 3 shows the topology of the simulation network. There are 50 sender nodes (s1-s50), one receiver (r1) and one destination node $(\mathrm{d} 1)$. The node $\mathrm{d} 1$ is investigated for its throughput and packet loss.

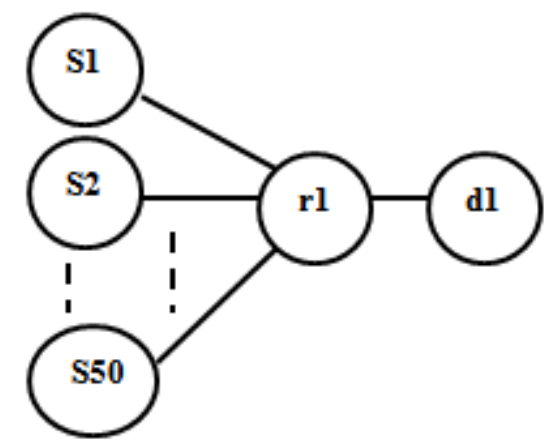

Fig 3: Network topology assumed.

Part of $t c l$ main program for simulation is listed below:

set ns [new Simulator] set NumSenders 50

set NumReceivers 1

\#read scenario, seed and bottleneck bandwidth from the command line input arguments

set Scenario [lindex \$argv 0]

set function [lindex \$argv 1]

set seed [lindex \$argv 2]

puts "scenario: \$Scenario; function: \$function; seed: \$seed" ns-random \$seed

set BufSize 100

set PktSize 1024

\#set winSize 200

\#in seconds

set Duration 50

\#create all nodes: note that the order of creating the nodes matter

for $\{$ set i 1$\}\{\$$ i $<=\$$ NumSenders $\}$ incr i $\}\{$

set $\mathrm{s}(\$ \mathrm{i})[\$ \mathrm{~ns}$ node] \}

set r1 [\$ns node]

set d1 [ $\$$ ns node]

\#open the nam trace file

set nf [open out.nam w]

\$ns namtrace-all \$nf

\#open the traffic trace file to record all events

set nd [open out5.tr w]

\$ns trace-all \$nd

\#define a finish procedure

proc finish \{\}\{

global ns nf nd qtf

\$ns flush-trace

close \$nf

close \$nd

close \$qtf

\#start nam

\#exec nam out.nam \&

exit 0

\}

$*$

$*$

for $\{$ set i 1$\}\{\$$ i $<=\$ N$ NumSenders $\}$ incr i $\}\{$ \$ns duplex-link \$s(\$i) \$r1 10Mb 100ms DropTail \$ns queue-limit \$s(\$i) \$r1 \$BufSize

\}

\#r1 d1 and d1 r1 are different

\$ns duplex-link \$r1 \$d1 5Mb 100ms RED

\$ns queue-limit \$r1 \$d1 \$BufSize

\#trace the queue: note that link $\mathrm{r} 1 \mathrm{~d} 1$ is different from $\mathrm{d} 1 \mathrm{r} 1$ set redq [[\$ns link \$r1 \$d1] queue]

set qtf [open queue.txt w]

\$redq trace curq_

\$redq trace ave_

\$redq attach \$qtf

\#setting up TCP connections

for $\{$ set i 1$\}\{\$$ i $<=\$$ NumSenders $\}$ incr $i\}\{$

set tcp $(\$ i)$ [new Agent/TCP]

\$ns attach-agent \$s $(\$ i)$ tcp $(\$ i)$

set $\operatorname{sink}(\$ i)$ [new Agent/TCPSink]

\$ns attach-agent $\$ \mathrm{~d} 1 \$ \operatorname{sink}(\$ \mathrm{i})$

$\$$ ns connect $\$$ tcp $(\$ i) \$ \operatorname{sink}(\$ \mathrm{i})$ 
$\$$ tcp $(\$ i)$ set fid_\$i

\$tcp $(\$ i)$ set packetSize_\$PktSize

\#\$tcp (\$i) set window_\$winSize

\#set up FTP over TCP connection as traffic source set $\mathrm{ftp}(\$ \mathrm{i})$ [new Application/FTP]

$\$ \mathrm{ftp}(\$ \mathrm{i})$ attach-agent $\$ \mathrm{tcp}(\$ \mathrm{i})$

$\$ \mathrm{ftp}(\$ \mathrm{i})$ set type FTP

\}

\#schedule events for the FTP agents

set StartTime [expr [ns-random] / 2147483647.0 / 100]

puts "starttime \$StartTime"

\#temporarily set to 2

for $\{$ set i 1$\}\{\$ \mathrm{i}<=\$ N$ umSenders $\}$ incr i $\}$ \{

\$ns at \$StartTime "\$ftp $(\$ i)$ start"

\$ns at \$Duration+\$StartTime "\$ftp(\$i) stop"

\}

\#ensure the ftp application have enough time to finish, so we $+1$

\$ns at \$Duration+\$StartTime+1 "finish"

\#run the simulation

\$ns run

\section{SIMULATION RESULTS}

\subsection{Queue Size}

From our simulation results, we can get the curves about current queue size and average queue size as in Fig 4. The 'drop probability' is selected based on the average queue size, not the current queue size.

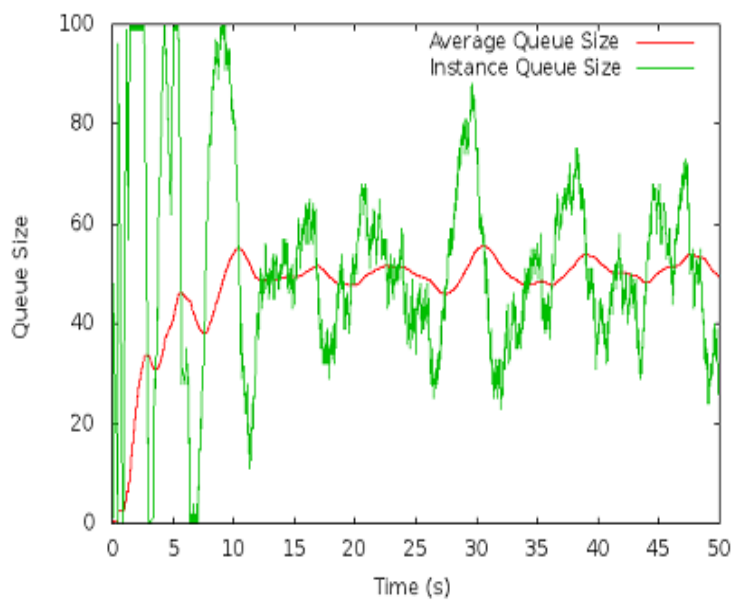

(a)

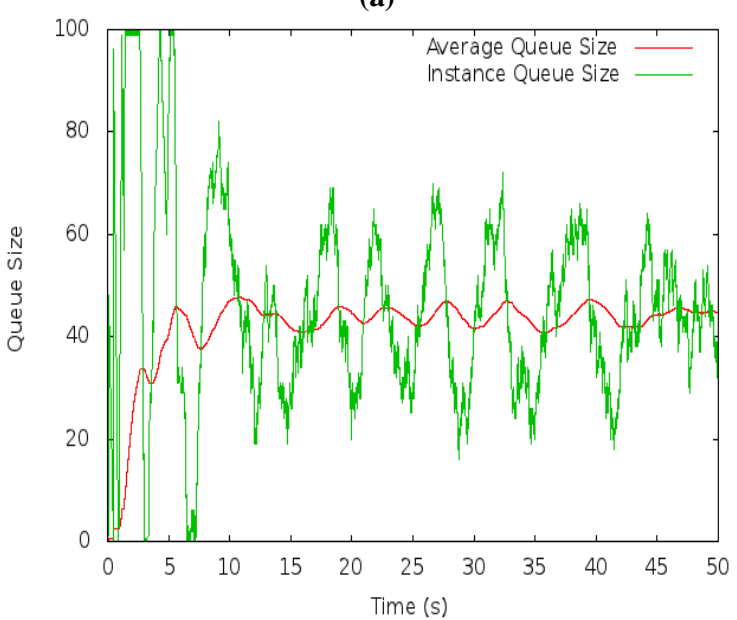

(b)

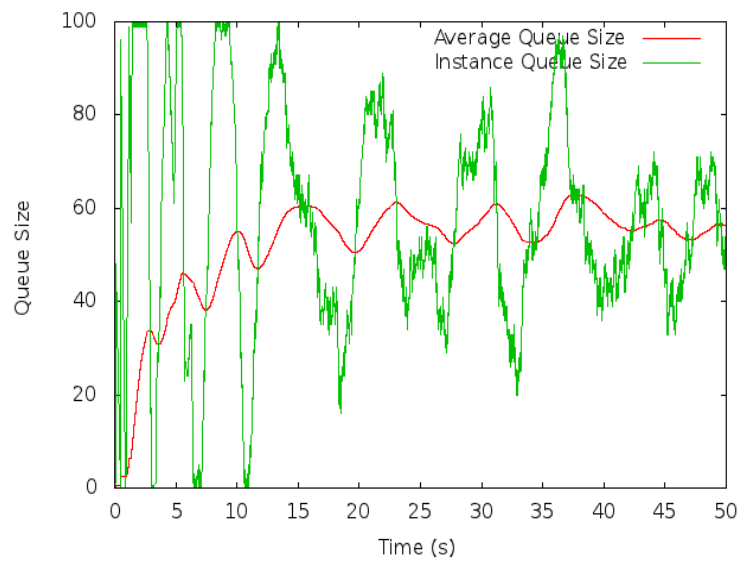

(c)

Fig 4: Queue size and average queue size of (a) RED (b) RED using V1 function (c) RED using V2 function.

From the plot, we can see that V1 keeps the average queue size at a lower level than regular RED, while V2 keeps the average queue size at a higher level than regular RED. This is expected as V1 is always above the original linear function given the same average queue size value in the range of $\left[\mathrm{th}_{\min }\right.$, $\mathrm{th}_{\max }$ ], in other words, V1 are more aggressive in terms of dropping packets when the average queue size is between th $_{\min }$ and $\mathrm{th}_{\max }$. On the contrast, V2 are less aggressive than regular RED.

\subsection{Packet loss}

The number of packet dropped in course of simulation period can be shown by Fig 5:

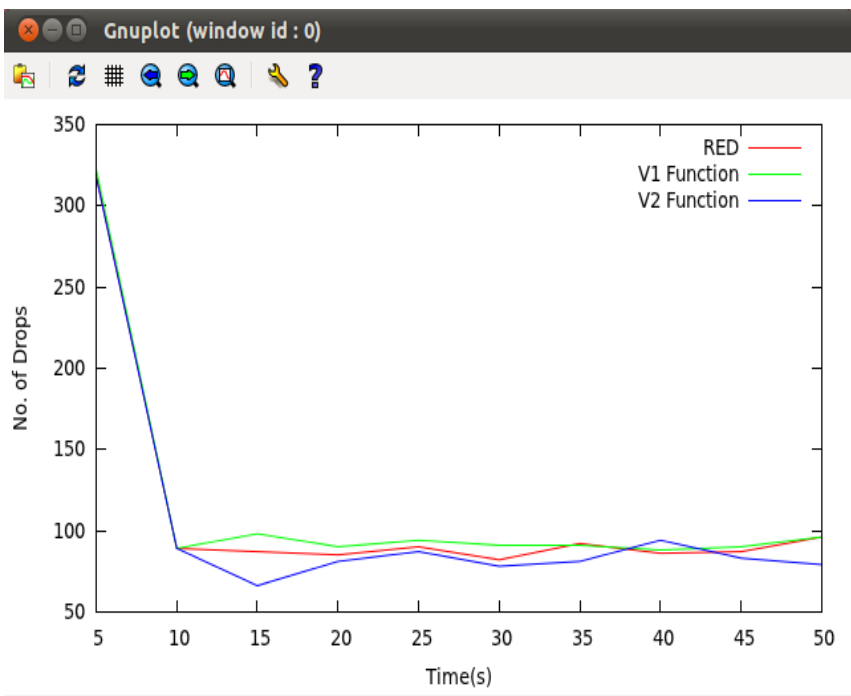

$51.8963,68.9323$

Fig 5: Pattern of packets dropped in assumed time duration

From the plot, we can see that V2 ensures less packet drop than regular RED and vice versa in case of V1 in which packet is dropped at a higher rate than V1 and regular RED algorithm.

\subsection{Throughput}

Throughput for both V1 and V2 function in comparison to RED can be clearly viewed in Fig 6: 


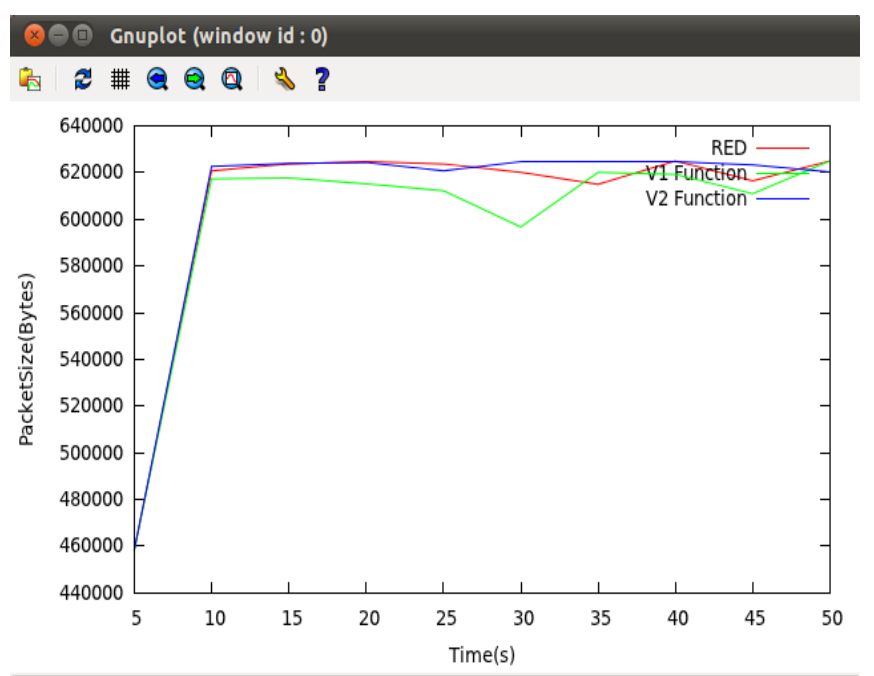

39.5932, 416924.

Fig 6: Throughput pattern in assumed time duration

From the plot, we can see that V2 function ensures higher throughput than regular RED and V1 function.

\section{CONCLUSIONS}

To avert the exploitation of bandwidth over the network various active queue management techniques have been introduced over the decade. In this paper my simulation result reflects the probable way of modifications in RED and their advantages over the regular concept of RED where packet loss can be considerably reduced. We have chosen a quadratic function (V2) showing convex pattern for drop probability and replacing $v \_a$ and $v \_b$, used in equation (3), with their new derived values $m$ and $n$ respectively. Therefore various possible convex functions for drop probability can be considered and their effect on various network traffics can be evaluated for enhancing the performance of the algorithm than conventional RED algorithm.

\section{REFERENCES}

[1] "Improving Internet Congestion Control and Queue Management Algorithms" by Wu-chang Feng. A dissertation submitted in partial fulfillment of the requirements for the degree of Doctor of Philosophy (Computer Science and Engineering) in The University of Michigan, 1999.

[2] S. Dijkstra, Modeling active queue management algorithms using stochastic Petri nets, Master's Thesis, University of Twente, 2004

[3] Lau, F.; Rubin, S.H.; Smith, M.H.; Trajkovic, L.; , "Distributed denial of service attacks," Systems, Man, and Cybernetics, 2000 IEEE International Conference, vol.3, no., pp.2275-2280 vol.3, 2000.

[4] Shu-Gang Liu; , "Simulation and evaluation of random early detection in congestion control," Machine Learning and Cybernetics, 2008 International Conference, vol.4, no., pp.2363-2368, 12-15 July 2008.

[5] Chengyu Zhu; Yang, O.W.W.; Aweya, J.; Ouellette, M.; Montuno, D.Y.; "A comparison of active queue management algorithms using the OPNET Modeler," Communications Magazine, IEEE, vol.40, no.6, pp.158167, June 2002

[6] CDouligeris, C.; Mitrokotsa, A.; "DDoS attacks and defense mechanisms: a classification," Signal Processing and Information Technology, 2003. ISSPIT 2003. Proceedings of the 3rd IEEE International Symposium, vol., no., pp. 190- 193, 14-17 Dec. 2003.Brown, L. D., Hua, H., and Gao, C. 2003.

[7] Stephen Specht \& Ruby Lee; "Taxonomies of Distributed Denial of Service Networks, Attacks, Tools, and Countermeasures"; May 2003.

[8] Changwang Zhang; Jianping Yin; Zhiping Cai; Weifeng Chen; , "RRED: robust RED algorithm to counter lowrate denial-of-service attacks," Communications Letters, IEEE , vol.14, no.5, pp.489-491, May 2010.

[9] Jae Chung; Claypool, M.; "Analysis of active queue management," Network Computing and Applications, 2003. NCA 2003. Second IEEE International Symposium, vol., no., pp. 359- 366, 16-18 April 2003.

[10] "Problem on modification of RED" by Richard T.B.Ma an Assistant Professor in School of Computing, National University of Singapore. 